Internet und Recht

Michael Müller-Brockhausen

Haftung für den Missbrauch von Zugangsdaten im Internet 


\section{Internet und Recht}

Herausgegeben von

Prof. Dr. Georg Borges

Universität des Saarlandes

Band 14 
Michael Müller-Brockhausen

\section{Haftung für den Missbrauch von Zugangsdaten im Internet}


Die Deutsche Nationalbibliothek verzeichnet diese Publikation in der Deutschen Nationalbibliografie; detaillierte bibliografische Daten sind im Internet über http://dnb.d-nb.de abrufbar.

Zugl.: Marburg, Univ., Diss., 2014

ISBN 978-3-8487-1576-3 (Print)

ISBN 978-3-8452-5591-O (ePDF)

1. Auflage 2014

(c) Nomos Verlagsgesellschaft, Baden-Baden 2014. Printed in Germany. Alle Rechte, auch die des Nachdrucks von Auszügen, der fotomechanischen Wiedergabe und der Übersetzung, vorbehalten. Gedruckt auf alterungsbeständigem Papier. 
Meiner Familie 
https://doi.org/10.5771/9783845255910_1

Generiert durch IP '172.22.53.54', am 26.04.2023, 02:37:35. 


\section{Vorwort}

Diese Arbeit wurde im Wintersemster 2013/2014 an der Philipps-Universität Marburg als Dissertation angenommen. Bei allen, die mich bei der Erstellung dieser Arbeit unterstützt haben, möchte ich mich an dieser Stelle herzlich bedanken.

Ein ganz besonderer Dank gebührt meinem Doktorvater Herrn Prof. Dr. Thomas Riehm. Ihm danke ich für exzellente Betreuung und die vielen hilfreichen Gespräche. Herrn Prof. Dr. Michael Kling bin ich dankbar für die zügige Erstellung des Zweitgutachtens. Für die Aufnahme in die Schriftenreihe möchte ich mich bei Herrn Prof. Dr. Georg Borges bedanken.

Herrn Dr. Matthias Schulz möchte ich herzlich danken für seine kritischen Gedanken zum Manuskript und die hilfreichen Diskussionen über juristische Probleme. Herrn Dr. Carsten Jungmann möchte ich neben seinen Anmerkungen zu einem Teil des Manuskripts insbesondere für alles danken, was ich von ihm gelernt habe.

Technisch habe ich die vorliegende Arbeit mit LATEX umgesetzt. Dabei schrieb ich viele Zeilen Quelltext selbst, um das gewünschte Ergebnis zu erreichen. Einige hilfreiche Vorlagen haben diese Arbeit deutlich erleichtert. Für die Klasse jurabook möchte ich mich bei Herrn Dr. Axel Sodtalbers bedanken. Der verwendete Zitierstil basiert auf biblatex-juradiss von Herrn Dr. Tobias Schwan, dem ich danken möchte. Herrn Audrey Boruvka, Mitautor von biblatex, gebührt Dank für die technische Hilfe bei der Lösung meiner speziellen Wünsche.

Meiner Familie, der diese Arbeit gewidmet ist, bin ich unendlich dankbar. Vielen Dank für die uneingeschränkte Förderung meiner Ausbildung und die liebevolle Unterstützung bei der Anfertigung der vorliegenden Arbeit. 
https://doi.org/10.5771/9783845255910_1

Generiert durch IP '172.22.53.54', am 26.04.2023, 02:37:35. 
Inhaltsübersicht

Abkürzungsverzeichnis

\begin{tabular}{ll|l}
$\$ 1$ & Einleitung & 31
\end{tabular}

\begin{tabular}{lll}
\hline Kapitel 1 & Technische und juristische Grundlagen & 39
\end{tabular}

$\$ 2$ Technische Grundlagen 39

$\begin{array}{lll}\S 3 & \text { Rechtsscheinhaftung } & 127\end{array}$

$\begin{array}{lll}\S 4 & \text { Der Vertragsschluss im Internet } & 147\end{array}$

Kapitel 2 Die Haftung für den Missbrauch von Zugangsdaten im Internet in unterschiedlichen Konstellationen 157

\$5 Haftung des Account-Inhabers bei bewusster Weitergabe der Zugangsdaten 157

\$ 6 Haftung des Account-Inhabers ohne bewusste Weitergabe der Zugangsdaten 193

$\S 7$ Haftung des Account-Inhabers bei Erstellen des Accounts durch Dritten 349

$\S 8$ Deliktische Haftung des Account-Inhabers 353

\$9 Haftung der anderen Beteiligten 371

$\S 10 \quad$ Beweiserleichterungen bei der Haftung für den Missbrauch von Zugangsdaten im Internet

Kapitel 3 Anwendung und Zusammenfassung der Ergebnisse 411 
$\S 11$ Anwendung der Ergebnisse auf verschiedene Account-Typen 411

$\S 12$ Zusammenfassung der Ergebnisse 453

\begin{tabular}{ll}
\hline Entscheidungsverzeichnis & 455
\end{tabular}

$\begin{array}{ll}\text { Literaturverzeichnis } & 465\end{array}$

$\begin{array}{ll}\text { Stichwortverzeichnis } & 495\end{array}$ 
Inhaltsverzeichnis

Abkürzungsverzeichnis

$\$ 1$ Einleitung 31

I. Problemaufriss 32

II. Zentrale Begriffe 36

1. Zugangsdaten 36

2. Missbrauch 37

3. Haftung 37

III. Gang der Darstellung 38

\begin{tabular}{lll}
\hline Kapitel 1 & Technische und juristische Grundlagen & 39
\end{tabular}

$\$ 2$ Technische Grundlagen 39

I. Internet 39

II. Zugangsdaten 40

1. Identiät 41

2. Identifikationsfunktion von Accounts im Internet 44

a) Internetzugang - IP-Adresse 45

aa) Internetanschluss 46

bb) WLAN 47

cc) IP-Adresse 47

b) E-Mail-Adresse 50

c) Passwortgeschützte Benutzerkonten auf Internetseiten 53

aa) Informationsportale 54

bb) eCommerce-Seiten, Online-Shops 55

cc) Internet-Auktionsplattformen mit Reputationssystem 56

d) Online-Banking 58 
e) Online-Bezahldienste 59

f) Elektronische Signatur 60

aa) Formen der elektronischen Signatur 61

bb) Asymmetrische Verschlüsselung 62

cc) Der Zertifizierungsdiensteanbieter als Trusted Authority 64

dd) Die Akzeptanz der elektronischen Signatur 65

ee) Exkurs: Ausblick 67

g) Elektronischer Identitätsnachweis im neuen Personalausweis (nPA) 68

h) De-Mail $\quad 70$

i) Zwischenergebnis zu den staatlichen Maßnahmen 74

3. Authentisierung, Authentifizierung und Autorisierung 74

a) Authentisierungsmittel 76

aa) Wissen 77

bb) Besitz 78

cc) Sein 80

b) Zwei- und Mehr-Faktor-Authentisierung 82

4. Besondere Merkmale von Zugangsdaten im Internet 84

$\begin{array}{ll}\text { III. Missbrauch } & 85\end{array}$

1. Missbrauch nach bewusster Weitergabe der $\mathrm{Zu}-$ gangsdaten 85

2. Missbrauch ohne bewusste Weitergabe der $\mathrm{Zu}-$ gangsdaten 86

a) Wege, um an die Zugangsdaten zu gelangen 89

aa) Physikalischer Zugriff auf die Zugangsdaten 89

bb) Zugriff zu gespeicherten Zugangsdaten 90

cc) Phishing 91

aaa) Klassisches Phishing 93

bbb) Pharming 95

ccc) Zweite Phase: die Internetseite des Angreifers 99

dd) Social Engineering 100

ee) Keylogger 102

ff) Man-in-the-Middle-Angriff (MitM-Angriff) 103 
gg) Sniffing: Mitlesen des Datenverkehrs

hh) Erraten der Zugangsdaten durch Ausprobieren bekannter Daten oder durch BruteForce-Angriffe

b) Infektionswege

aa) Sicherheitslücken in Programmen, ZeroDay-Exploits

bb) Computervirus

cc) Computerwurm

dd) Trojanisches Pferd, Trojaner

ee) Rootkits

114

ff) Drive-By-Infection

114

c) Schutz gegen Infektionen des Rechners

aa) Antiviren-Programm

bb) Firewall

118

3. Missbrauch durch Erstellen eines Accounts unter falschem Namen

4. Missbrauch ohne Erlangen der Zugangsdaten vom Account-Inhaber

a) Mail-Spoofing

b) Schwachstellen beim Authentisierungsnehmer

aa) SQL-Injection

bb) Cross-Site-Scripting (XSS)

cc) Schwachstellen in der IT-Infrastruktur

dd) Unbefugte Weitergabe der Zugangsdaten

$\S 3$ Rechtsscheinhaftung

I. Voraussetzungen einer Rechtsscheinhaftung 127

1. Rechtsscheintatbestand 128

2. Zurechenbarkeit 131

a) Veranlassungsprinzip 132

b) Verschuldensprinzip 133

c) Risikoprinzip 136

d) Voraussetzungen und Fälle der Zurechnung 137

3. Schutzwürdigkeit des Geschäftsgegners 139 


\section{Disposition im Vertrauen auf den Rechtsschein 140 \\ II. Rechtsfolge der Rechtsscheinhaftung 141 \\ 1. Positives Interesse 141 \\ 2. Anfechtung des Rechtsscheins: negatives Interesse 141 \\ 3. Wahlrecht zwischen Schein und Wirklichkeit 142 \\ III. Beispiele für Rechtsscheinhaftung 142 \\ 1. Duldungsvollmacht 143 \\ 2. Anscheinsvollmacht 144}

$\$ 4$ Der Vertragsschluss im Internet 147

I. Vertragsschluss im Internet 148

II. Handeln unter fremdem Namen 150

1. Allgemein 150

2. Im Internet 151

III. Zwei- und Drei-Personen-Konstellationen 155

Kapitel 2 Die Haftung für den Missbrauch von Zugangsdaten im Internet in unterschiedlichen Konstellationen

$\S 5$ Haftung des Account-Inhabers bei bewusster Weitergabe der Zugangsdaten

I. $\quad$ Begriff der Weitergabe 158

II. Lösung über die Duldungsvollmacht 159

1. Bildschirmtext (Btx) 161

2. Kritik 162

III. Lösung über die Übertragung des Rechtsgedankens des § 172 Abs. 1 BGB 162

1. Ursprünglicher Anwendungsbereich des $§ 172$ Abs. 1 BGB

a) Bedeutung des § 172 Abs. 1 BGB 163

b) Auslegung des §172 Abs. 1 BGB 164

aa) Rechtsscheintatbestand $\quad 164$

bb) Zurechenbarkeit 167

cc) Disposition im Vertrauen auf den Rechtsschein 170

$\begin{array}{ll}\text { dd) Gutgläubigkeit des Dritten } & 170\end{array}$ 
2. Anwendung des $\S 172$ Abs. 1 BGB auf den Missbrauch von Zugangsdaten

3. Analoge Anwendung des $\S 172$ Abs. 1 BGB auf verdeckte Blanketterklärungen

a) Exkurs: Voraussetzungen einer analogen Anwendung

173

b) Erster Schritt: offene Blanketterklärungen

175

c) Zweiter Schritt: verdeckte Blanketterklärungen

176

d) Kein dritter Schritt: Der Kreditkartenmissbrauch 179

e) Analoge Anwendung des $\S 172$ Abs. 1 BGB auf den Missbrauch von Zugangsdaten im Internet 180

aa) Rechtsscheintatbestand

bb) Zurechenbarkeit

f) Zwischenergebnis

4. Zwischenergebnis

IV. Zwischenergebnis

\$6 Haftung des Account-Inhabers ohne bewusste Weitergabe

\begin{tabular}{|c|c|c|}
\hline \multicolumn{2}{|c|}{ der Zugangsdaten } & 193 \\
\hline I. & Lösung über die Anscheinsvollmacht & 193 \\
\hline & 1. Rechtsscheintatbestand & 194 \\
\hline & a) Sicherheitsstandard im Internet & 194 \\
\hline \multicolumn{3}{|c|}{ b) Handeln eines Dritten von gewisser Dauer und } \\
\hline & Häufigkeit & 196 \\
\hline & c) Identifikationsfunktion & 199 \\
\hline & d) Risikoverteilung & 200 \\
\hline & e) Keine Zurechnung nach deliktischen Grundsätzen & 203 \\
\hline & f) Zwischenergebnis & 204 \\
\hline & 2. Zurechenbarkeit & 204 \\
\hline & 3. Zwischenergebnis & 206 \\
\hline \multirow[t]{3}{*}{ II. } & Lösung über vorhandene vertragliche Beziehungen & 207 \\
\hline & 1. In Zwei-Personen-Konstellationen: Vertrag als & \\
\hline & Grundlage & 207 \\
\hline & 2. In Drei-Personen-Konstellationen: Schuldverhält- & \\
\hline
\end{tabular}


a) Bestehendes Vertragsverhältnis des AccountInhabers zu einem Diensteanbieter

b) Leistungsnähe des Dritten

c) Schutzwürdige Interessen des Gläubigers 214

d) Erkennbarkeit für den Schuldner 216

e) Schutzbedürftigkeit des Dritten 217

f) Umfang der Haftung 218

g) Zwischenergebnis 219

III. Lösung über die culpa in contrahendo 219

1. Allgemein zur culpa in contrahendo (c.i.c.) 220

2. Subsidiäre Anwendung der culpa in contrahendo? 222

3. Vorvertragliches Schuldverhältnis 223

4. Pflichtverletzung 228

a) Verhalten des Account-Inhabers 228

b) Verhaltenszurechnung als Anknüpfungspunkt? 232

5. Verschulden 233

6. Umfang der Haftung 235

7. Konkurrenzen 236

8. Zwischenergebnis 236

IV. Lösung über eine analoge Anwendung des § 122 BGB 237

1. Fehlendes Erklärungsbewusstsein 237

2. Abhandengekommene Willenserklärung 239

3. Anwendung im Internet 240

V. Lösung über das Deliktsrecht 243

1. §823 Abs. 1 BGB 243

2. \$823 Abs. 2 BGB 244

VI. Lösung über die allgemeinen Rechtsscheingrundsätze 244

1. Blick auf Rechtsscheintatbestände in vergleichbaren Fallkonstellationen

a) Vollmachtsurkunde, §172 Abs. 1 BGB 245

b) Briefpapier, Logos und Stempel 246

c) Rechtsscheinhaftung bei der Benutzung von Bildschirmtext (Btx) 249

aa) Rechtsscheintatbestand 249

bb) Zurechenbarkeit 251 
d) Bankgeschäfte 253

aa) Fehlerhafte Überweisungen 253

bb) ec-Karte 255

cc) Online-Banking 256

dd) Kreditkarte im Mail-Order-Verfahren 258

e) Haftung nach § 45i Abs. 4 S. 1 TKG 259

f) Zwischenergebnis 262

2. Rechtsscheintatbestand 263

a) Grundsätzliche Eignung 263

b) Sicherheit der verwendeten Authentisierungsmethoden 265

aa) Ohne Authentisierung 268

bb) Rein wissensbasierte Authentisierung 269

aaa) Sicherheit von Passwörtern durch ihre Stärke 270

bbb) Ausspähen von Passwörtern 272

ccc) Sicherung durch den Account-Inhaber 274

ddd) Sicherung durch den Authentisierungsnehmer 278

eee) Sicherheit der Kommunikation 281

fff) Schlussfolgerung für den Rechtsscheintatbestand 282

cc) Zwei-Faktor-Authentisierung 283

aaa) Sicherheit der Zwei-Faktor-Authentisierung 283

bbb) Missbrauchsmöglichkeiten bei der Zwei-Faktor-Authentisierung 284

ccc) Sicherung durch den Account-Inhaber 285

ddd) Sicherung durch den Authentisierungsnehmer 286

eee) Sicherheit der Kommunikation 287

fff) Schlussfolgerung für den Rechtsscheintatbestand 287

dd) Zwischenergebnis 288

c) Identifikationsfunktion von Accounts im Internet 288 
aa) Ohne Angabe von Personendaten 290

bb) Ohne Überprüfung der Personendaten 291

cc) Plausibilitätskontrolle der Personendaten 293

dd) Überprüfung der Personendaten 294

ee) Sicherstellung der Identität durch ein Reputationssystem 300

ff) Individuelle Überprüfung durch persönlichen Kontakt zum Account-Inhaber 302

gg) Zwischenergebnis 302

d) Angemessene Verteilung der Risiken 303

aa) Die vermeintliche Notwendigkeit Schutzbehauptungen zu verhindern 305

bb) Rechtsökonomisch sinnvolle Verteilung der Risiken 307

aaa) Die vier rechtsökonomischen Voraussetzungen der Vertrauenshaftung 308

(1) Asymmetrische Verteilung der Informationskosten 309

(2) Produktivität der Information 311

(3) Existenz einer Vertrauensprämie 313

(4) Höhe der Opportunismusprämie im Vergleich zur Vertrauensprämie 314

(5) Zwischenergebnis 315

bbb) Die Ausgestaltung einer Haftung aus rechtsökonomischer Sicht 315

cc) Alternative Möglichkeiten der Absicherung gegen Missbrauch 316

dd) Zwischenergebnis 320

e) Widerspruch zur herrschenden Ansicht bei Weitergabe der Zugangsdaten 320

f) Zwischenergebnis 322

3. Zurechenbarkeit 322

a) Möglichkeit den Rechtsschein zu zerstören 323

b) Beschränkung auf grobe Fahrlässigkeit? 324

c) Maßstab der Zurechnung 327 
d) Fälle der Zurechnung

aa) Sorgfalts- und Verkehrspflichten des Account-Inhabers

bb) Einzelfälle

e) Zwischenergebnis

4. Schutzwürdigkeit des Geschäftsgegners 344

5. Disposition im Vertrauen auf den Rechtsschein 344

6. Rechtsfolge

7. Zwischenergebnis

VII. Zwischenergebnis

$\S 7$ Haftung des Account-Inhabers bei Erstellen des Accounts durch Dritten

$\$ 8$ Deliktische Haftung des Account-Inhabers 353

I. Eigener Zurechnungstatbestand

II. Keine überzeugende dogmatische Begründung und Begründbarkeit

1. Fehlender Schutzzweckzusammenhang

2. Dogmatische Unstimmigkeiten

3. Möglichkeit der Herleitung über andere Normen, die Verhalten zurechnen

a) Verhaltenszurechnung bei Pflichtverletzungen in Sonderverbindungen

b) Verhaltenszurechnung bei der Haftung des Unternehmensinhabers

4. Herleitung des Unterlassungsanspruches aus § 1004 Abs. 1 BGB

5. Zwischenergebnis 364

III. Zweifelhafte Identifikationsfunktion 364

IV. Ausgestaltung einer möglichen Geheimhaltungspflicht 365

V. Belastung des Account-Inhabers 368

\begin{tabular}{lll}
\hline VI. Zwischenergebnis & 369
\end{tabular}

\$ 9 Haftung der anderen Beteiligten 371

I. Haftung des Handelnden 371 
1. Haftung gegenüber dem Geschäftsgegner 371

2. Haftung gegenüber dem Account-Inhaber 373

II. Haftung des Authentisierungsnehmers 373

$\$ 10$ Beweiserleichterungen bei der Haftung für den Missbrauch

\begin{tabular}{|c|c|c|}
\hline \multicolumn{2}{|c|}{ von Zugangsdaten im Internet } & \multirow{2}{*}{$\begin{array}{l}377 \\
378\end{array}$} \\
\hline I. & Formen der Beweiserleichterung & \\
\hline \multicolumn{3}{|c|}{ 1. Beweislastumkehr mit und ohne tatsächlicher Ver- } \\
\hline & mutung & 378 \\
\hline & a) Umkehr der Beweislast & 379 \\
\hline & b) Tatsächliche Vermutung & 382 \\
\hline & 2. Anscheinsbeweis & 385 \\
\hline & 3. Sekundäre Darlegungslast & 389 \\
\hline II. & \multicolumn{2}{|c|}{ Schutzbehauptungen durch freie richterliche Beweis- } \\
\hline & würdigung verhindern & 391 \\
\hline \multirow[t]{7}{*}{ III. } & \multicolumn{2}{|c|}{ Anerkannte Beweiserleichterungen in ähnlichen Kon- } \\
\hline & stellationen & 393 \\
\hline & 1. Elektronische Signatur & 393 \\
\hline & 2. $\quad$ Bildschirmtext $(\mathrm{Btx})$ & 398 \\
\hline & 3. ec-Karte & 400 \\
\hline & 4. Online-Banking & 404 \\
\hline & 5. Zwischenergebnis & 407 \\
\hline
\end{tabular}

Kapitel 3 Anwendung und Zusammenfassung der Ergebnisse 411

\$11 Anwendung der Ergebnisse auf verschiedene Account-Typen 411 I. Internetanschluss, IP-Adresse 411

1. Rechtsscheinhaftung 411

2. Beweiserleichterungen 412

II. E-Mails 413

1. Rechtsscheinhaftung 413

2. Beweiserleichterungen 414

III. Benutzerkonten auf Internetseiten 420

1. Rechtsscheinhaftung 420

a) Informationsportale und Online-Shops 420 
b) Internet-Auktionsplattformen

c) Accounts mit Zwei-Faktor-Authentisierung 423

2. Beweiserleichterungen

a) Anscheinsbeweis $\quad 424$

b) Sekundäre Darlegungslast $\quad 434$

IV. Online-Banking 435

V. Online-Bezahldienste 436

VI. Elektronische Signatur 437

1. Rechtsscheinhaftung 437

a) Sicherheit der Authentisierungsmethode 437

b) Zuverlässigkeit der Identifikationsfunktion 439

c) Zwischenergebnis 440

2. Beweiserleichterungen 441

VII. Elektronischer Identitätsnachweis 442

1. Rechtsscheinhaftung 442

a) Sicherheit der Authentisierungsmethode 442

b) Zuverlässigkeit der Identifikationsfunktion 444

2. Beweiserleichterungen 445

VIII. De-Mail 449

1. Rechtsscheinhaftung 449

2. Beweiserleichterungen 451

$\S 12$ Zusammenfassung der Ergebnisse 453

\begin{tabular}{ll}
\hline Entscheidungsverzeichnis & 455
\end{tabular}

\begin{tabular}{ll}
\hline Literaturverzeichnis & 465
\end{tabular}

Stichwortverzeichnis 495 
https://doi.org/10.5771/9783845255910_1

Generiert durch IP '172.22.53.54', am 26.04.2023, 02:37:35. 
a.A.

AcP

AG

AGB

AGB/B

Anh

Anm.

AnwBl

API

APWG

ARD

ARP

Art.

AT

$\mathrm{AO}$

BAG

BB

BDSG

BeurkG

BeckRS

Beil.

BGB

BGB1

BGH

BGHZ

BKA

BKR

BPG

BSI

BSIG
Andere Ansicht

Archiv für die civilistische Praxis

Amtsgericht

Allgemeine Geschäftsbedingungen

Allgemeine Geschäftsbedingungen der privaten Banken, AGB Banken

Anhang

Anmerkung

Anwaltsblatt

Application Programming Interface, zu deutsch

Programmierschnittstelle

Anti-Phishing Working Group

Arbeitsgemeinschaft der öffentlich-rechtlichen

Rundfunkanstalten der Bundesrepublik Deutschland

Address Resolution Protocol

Artikel

Allgemeiner Teil

Abgabenordnung

Bundesarbeitsgericht

Betriebsberater

Bundesdatenschutzgesetz

Beurkungdungsgesetz

Beck Rechtsprechung

Beilage

Bürgerliches Gesetzbuch

Bundesgesetzblatt

Bundesgerichtshof

Entscheidungen des Bundesgerichtshofs in Zivilsachen

Bundeskriminalamt

Zeitschrift für Bank- und Kapitalmarktrecht

Bürgerportalgesetz

Bundesamt für Sicherheit in der Informationstechnik

Gesetz über das Bundesamt für Sicherheit in der

Informationstechnik (BSI-Gesetz) 
BT Bundestag / Besonderer Teil

Btx Bildschirmtext

BVerfG Bundesverfassungsgericht

BVerfGE Entscheidungen des Bundesverfassungsgerichts

CD Compact Disc

c.i.c. culpa in contrahendo

CMS Content Management System

CPU Central Processing Unit

CR Computer und Recht

c't Magazin für Computertechnik

DIN Deutsche Industrienorm

DJT Deutscher Juristentag

DeMailG Gesetz zur Regelung von De-Mail-Diensten (De-MailGesetz)

Denic Deutsches Network Information Center eG

DNA deoxyribonucleic acid, deutsch Desoxyribonukleinsäure (DNS)

DNS Domain Name System

DNSSEC Domain Name System Security Extensions

DSL Digital Subscriber Line

DuD Datenschutz und Datensicherheit (Zeitschrift)

DÖV Die Öffentliche Verwaltung (Zeitschrift)

DZWir Deutsche Zeitschrift für Wirtschaftsrecht

ec Electrnonic Cash

eCommerce Electronic Commerce, auf Deutsch elektronischer

Geschäftsverkehr

eG Eingetragene Genossenschaft

eID electronic identity

E-Mail Electronical Mail, auf Deutsch elektronische Post

EG Europäische Gemeinschaft

EWiR Entscheidungen zum Wirtschaftsrecht

FG Festgabe

FormAnpG Gesetz zur Anpassung der Formvorschriften des

Privatrechts und anderer Vorschriften an den modernen

Rechtsgeschäftsverkehr (Formanpassungsgesetz)

FTP File Transfer Protocol

FS Festschrift

GG Grundgesetz 
GmbH Gesellschaft mit beschränkter Haftung

GRUR Gewerblicher Rechtsschutz und Urheberrecht (Zeitschrift)

GRUR-RR Gewerblicher Rechtsschutz und Urheberrecht

Rechtsprechungs-Report (Zeitschrift)

GSM

Global System for Mobile Communications

GUID

Global Unique Identifier

GwG

Gesetz über das Aufspüren von Gewinnen aus schweren

Straftaten, (Geldwäschegesetz)

HBCI Homebanking Computer Interface

HGB Handelsgesetzbuch

HTTP

Hypertext Transfer Protocol

ICANN Internet Corporation for Assigned Names and Numbers

i.e.S. im engeren Sinn

IEEE Institute of Electrical and Electronics Engineers

IETF Internet Engineering Task Force

iFrame Inlineframe

IMAP Internet Message Access Protocol

IMSI

International Mobile Subscriber Identity

Internet Interconnected Networks

IP

Internet Protocol

IPv4

Internet Protocol Version 4

IPv6

ISP

Internet Protocol Version 6

Internet Service Provider

IT

ITR

Informationstechnik

ITRB

IT-Recht

iTAN

Der IT-Rechtsberater

IuKDG

Indizierte Transaktionsnummer

JA

Informations- und Kommunikationsdienste-Gesetz

JherJB

Juristische Arbeitsblätter

$\begin{array}{ll}\text { JherJB } & \begin{array}{l}\text { Jahrbücher für die Dogmatik des heutigen römischen und } \\ \text { deutschen Rechts (Jhering-Jahrbücher) }\end{array} \\ \text { JKomG } & \begin{array}{l}\text { Justizkommunikationsgesetz } \\ \text { JR }\end{array} \\ \text { Juristische Rundschau } \\ \text { Juristische Ausbildung } \\ \text { JurisPR } & \text { Juris-Praxisreport } \\ \text { JurPC } & \text { Internet-Zeitschrift für Rechtsinformatik und } \\ & \text { Informationsrecht } \\ \text { JuS } & \text { Juristische Schulung }\end{array}$


JW Juristische Wochenschrift

JZ Juristenzeitung

Habil. Habilitation

HTML Hypertext Markup Language

KG Kammergericht

K\&R Kommunikation und Recht

KOM Kommission

LAN Local Area Network

LG Landgericht

lit. Litera

LZ Leipziger Zeitschrift für Deutsches Recht

LMK Lindenmaier-Möhring - Kommentierte BGH-Rechtsprechung

Losebl. Loseblatt-Sammlung

MarkenG Markengesetz

MDR Monatsschrift für Deutsches Recht

MittBayNot Mitteilungen des Bayerischen Notarvereins, der Notarkasse und der Landesnotarkammer Bayern

MitM Man-in-the-Middle

NK NomosKommentar

MMR Multimedia und Recht

mTAN Mobile TAN

MUA Mail User Agent, auch als E-Mail-Programm, E-MailClient bezeichnet

MüKo Münchener Kommentar

MTA Mail Transfer Agent

m.w.N. mit weiteren Nachweisen

NIC Network Information Center

NJ Neue Justiz

NJOZ Neue Juristische Online-Zeitschrift

NJW Neue Juristische Wochenschrift

NJW-CoR Computerreport der Neuen Juristischen Wochenschrift

NJW-RR Neue Juristische Wochenschrift, Rechtsprechungs-Report

nPA neuer Personalausweis

NStZ Neue Zeitschrift für Strafrecht

NZV Neue Zeitschrift für Verkehrsrecht

OHG Offene Handelsgesellschaft

OLG Oberlandesgericht 
OLG-NL OLG-Rechtsprechung neue Länder

OLGZ Entscheidungsammlung der Oberlandesgerichte in

Zivilsachen einschließlich der freiwilligen Gerichtsbarkeit

PAuswG Personalausweisgesetz

PAuswV Personalausweisverordnung

PIN Persönliche Identifikationsnummer

PGP Pretty Good Privacy

PKI Public-Key-Infrastruktur

POP3 Post Office Protocol, Version 3

POS Point of Sale

PostIdent Identifikationsverfahren als Dienstleitung der Deutschen Post AG

provet Projektgruppe verfassungsverträgliche Technikgestaltung

RFC Request for Comments, deutsch: Bitte um Kommentare

RFID radio-frequency identification

RG Reichtsgericht

RGRK Reichsgerichtsräte-Kommentar

RGZ Entscheidungen des Reichsgerichts in Zivilsachen

Rn. Randnummer

RPfleger Der Deutsche Rechtspfleger

SB Sonderbedingungen

ScheckG Scheckgesetz

Schr. Schrift

Schufa Schutzgemeinschaft für allgemeine Kreditsicherung, mittlerweile als Schufa Holding AG tätig

1. SigÄndG Erstes Gesetz zur Änderung des Signaturgesetzes

SigG Signaturgesetz

SigV Verordnung zur elektronischen Signatur

SIM Subscriber Identity Module

SMG Gesetz zur Modernisierung des Schuldrechts

(Schuldrechtsmodernisierungsgegsetz)

SMS Short Message Service

SMTP Simple Mail Transfer Protocol

SSL Secure Sockets Layer

SSO Single Sign-on

StGB Strafgesetzbuch

StPO Strafprozessordnung

StVG Straßenverkehrsgesetz 
StVO Straßenverkehrsordnung

SQL Structured Query Language

TAN Transaktionsnummer

TCP Transmission Control Protocol

TKG Telekommunikationsgesetz

TKV Telekommunikations-Kundenschutzverordnung

TLD Top-Level-Domain

u.d.T. unter dem Titel

UFITA Archiv für Urheber-, Film-, Funk- und Theaterrecht

ULD Unabhängiges Landeszentrum für Datenschutz SchleswigHolstein

UMTS Universal Mobile Telecommunications System

Univ. Universität

URL Uniform Resource Locator

USA United States of America, deutsch Vereinigte Staaten von Amerika

USB Universal Serial Bus

USD US Dollar

UStG Umsatzsteuergesetz

UStDV Umsatzsteuer-Durchführungsverordnung

UrhG Urhebergesetz

UWG Unlautererwettbewerbsgesetz

VersR Versicherungsrecht (Zeitschrift)

WechselG Wechselgesetz

WRP Wettbewerb in Recht und Praxis

WLAN Wireless LAN

Web-Dok. Web-Dokument

WEP Wired Equivalent Privacy

WPA Wi-Fi Protected Access

WM Wertpapier-Mitteilungen, Zeitschrift für Wirtschafts- und Bankrecht

WuM Wohnungswirtschaft und Mietrecht, Zeitschrift

WWW World Wide Web

XSS Cross-Site-Scripting

ZAG Zahlungsdiensteaufsichtsgesetz

ZD Zeitschrift für Datenschutz

ZDF Zweite Deutsche Fernsehen 
ZDRL Zahlungsdienste-Richtlinie, Richtlinie 2007/64/EG über Zahlungsdienste im Binnenmarkt

ZHR Zeitschrift für das gesamte Handelsrecht und Wirtschaftsrecht

ZIP Zeitschrift für Wirtschaftsrecht, früher: Insolvenzrecht Zeitschrift für die gesamte Insolvenzpraxis

ZPO Zivilprozessordnung

ZUM Zeitschrift für Urheber- und Medienrecht

ZZP Zeitschrift für Zivilprozeß 
https://doi.org/10.5771/9783845255910_1

Generiert durch IP '172.22.53.54', am 26.04.2023, 02:37:35. 